\title{
Comparación de dosis de morfina en bloqueos caudales en cirugías traumatológicas de alto impacto
}

Torres Rivas LP. ${ }^{1}$, Neivert FJJ. ${ }^{2}$, Alzugaray E. ${ }^{2}$, Sanz GA. ${ }^{2}$, Andersson SM. ${ }^{2}$, Caliva JA. ${ }^{2}$, Roses MG. ${ }^{1}$, Harmut CF. ${ }^{1}$, Izasiz E. ${ }^{1}$, Pérez G. ${ }^{1}$, Cibils M. ${ }^{1}$

1 Hospital Provincial de Pediatría Dr. Fernando Barreyro, Posadas, Misiones, Argentina.

2 Hospital de Agudos Dr. Ramón Madariaga, Posadas, Misiones, Argentina.

Introducción: El bloqueo caudal es una de las técnicas de anestesia regional pediátrica más utilizadas, de fácil realización, bajo coste y que proporciona una excelente analgesia postoperatoria.

La duración de la analgesia con bupivacaína en conjunto con morfina se extiende hasta $24 \mathrm{~h}$ con aparición de efectos adversos asociados al opioide. Estudios demuestran que podrían administrarse dosis menores de opioides brindando analgesia y disminuyendo las reacciones adversas.

Métodos: Criterios de inclusión: Pacientes $<$ de 9 años, con $<30 \mathrm{~kg}$, de ambos sexos, sometidos a cirugías traumatológicas de miembros inferiores de alto impacto.

Criterios de exclusión: Pacientes con malformaciones de la columna vertebral, antecedentes de cirugía del canalmedular, enfermedades degenerativas con o sin secuelas, infecciones de la piel en zona apunzar, coagulopatía e hipovolemia.

Premedicación: Cefazolina $25 \mathrm{mg} / \mathrm{kg}$, dexametasona 0,1 mg/kg e ibuprofeno $10 \mathrm{mg} / \mathrm{kg}$.

Anestesia general: Inducción: sevofluorano + fentanilo $2 \mathrm{mcg} / \mathrm{kg}$. Mantenimiento: sevofluorano + remifentanilo 0,5 $\mathrm{mcg} / \mathrm{kg}$

Grupo 1: Bupivacaína isobárica al 0,25\%;0,5 ml/kg y morfina $<30 \mathrm{mcg} / \mathrm{kg}$.

Grupo 2: Bupivacaína isobárica al 0,25\%; 0,5 ml/kg y morfina de 30-50 mcg/kg.

Plan postoperatorio: Ibuprofeno $10 \mathrm{mg} / \mathrm{kg}$ cada $8 \mathrm{~h}$ y rescate analgésico con tramadol $1 \mathrm{mg} / \mathrm{kg}$.

Se evaluará el dolor al despertar, a las 2, 6, 12 y 24 h; en niños de 1-36 meses con la escala de FLACC, de 3-7 años con la escala de caras de Wong- Baker (Figura 1) y a los mayores de 8 años con la escala numérica.

Se registrará la aparición de sedación, retención urinaria, náuseas y vómitos, si se requirió alguna intervención para revertirlos y la cantidad de días de internación.

Análisis estadístico de tipo descriptivo.

Resultados: Se realizó en 31 pacientes, 18 femeninos y 13 masculinos, con un promedio de edad de 49,22 meses, con un desvío estándar \pm 19,64; 16 pertenecen al grupo 1 y 15 al grupo 2. Se obtuvieron los siguientes resultados: (Figura. 2 y Tabla 1).

Conclusiones: Una cobertura analgésica adecuada con dosis bajas de opioides requiere dosis estándar de anestésicos locales. A partir de $25 \mathrm{mcg} / \mathrm{kg}$ de morfina vía caudal se presentó menor incidencia de dolor postoperatorio y de estancia hospitalaria, sin aumentar la frecuencia de retención urinaria ni sedación. Dosis menores a $30 \mathrm{mcg} / \mathrm{kg}$ no mostró menor aparición de náuseas y vómitos. La retención urinaria fue más reiterada a partir de $\operatorname{los} 30 \mathrm{mcg} / \mathrm{kg}$ y la sedación a partir de los $40 \mathrm{mcg} / \mathrm{kg}$. Este estudio podría considerarse como preliminar, siendo necesario un mayor número de determinaciones.

https://doi.org/10.25237/congresoclasa2019.73 